\title{
Zwangsmaßnahmen gegen den Iran im Lichte der Sanktions-Forschung
}

\author{
Bernd W. Kubbig \\ (in Zusammenarbeit mit Sven-Eric Fikenscher)
}

\begin{abstract}
This article applies major findings of the international research on sanctions to the current boycott and disinvestment strategies being applied against the Islamic Republic of Iran. The key findings of this research include the identification of three conditions of success for coercive activities: the coherence of the multilateral alliance, the vulnerability of the target country, and the determination of the penalizing states to bear even high economic costs. The main empirical focus of the paper is on the United Nations' sanctions as codified in UN Security Council Resolutions 1737 and 1747. It also deals with the comprehensive disinvestment campaign of the George W. Bush administration. The paper is sceptical regarding the success of the sanctions that have been implemented, stressing that all three conditions have so far only been fulfilled to a limited extent.
\end{abstract}

Keywords: Sanktionen, UN-Sicherheitsrat, US-amerikanische Außenpolitik, Islamische Republik Iran, Nichtverbreitung.

\section{1. (Wann) können Strafmaßnahmen gegen den Iran wirksam sein?}

$\mathrm{N}$ ach jahrelangen Debatten beschloss der Gouverneursrat der Internationalen Atomenergie-Organisation (IAEO) am 4. Februar 2006, den Weltsicherheitsrat mit dem Disput über das iranische Nuklearprogramm zu betrauen. Am 23. Dezember 2006 und am 24. März 2007 verabschiedete dieses Gremium mit den Resolutionen 1737 und 1747 wirtschafts-, finanz- und reisepolitische Maßnahmen gegen Teheran. Über eine dritte Entschließung wird im UN-Sicherheitsrat debattiert. Unter welchen Bedingungen lässt sich die Islamische Republik durch Sanktionen zur Aufgabe der Urananreicherung zwingen? Das ist die Forschungsfrage dieses Aufsatzes. Teherans Verzicht auf diesem Gebiet ist gleichzeitig das vorrangige politische Ziel der UN-Resolutionen.

Zur Beantwortung dieser Frage destilliert der Beitrag vier Resultate aus der Sanktions-Forschung, die als relativ gesichert gelten können: ${ }^{2}$

1. die überwiegende Erfolglosigkeit unilateraler Zwangsmaßnahmen;

2. ein differenzierter Sanktionsbegriff, der zwischen umfassenden und gezielten Maßnahmen unterscheidet;

3. drei zentrale Erfolgsbedingungen für internationale Zwangsmaßnahmen - nämlich große Geschlossenheit der Sanktions-Allianz, hoher Grad der ökonomischen Verwundbar-

Referierter Beitrag.

1 PD Dr. Bernd W. Kubbig ist Projektleiter an der Hessischen Stiftung Friedensund Konfliktforschung (HSFK) und Privatdozent an der Johann Wolfgang Goethe-Universität in Frankfurt/M. Sven-Eric Fikenscher ist studentischer Mitarbeiter der HSFK-Projektgruppe Raketenabwehrforschung International. - Dieser Beitrag beruht in wesentlichen Teilen auf: Bernd W. Kubbig (in Zusammenarbeit mit Sven-Eric Fikenscher), Internationale Sanktionen gegen den Iran: Erfolgsbedingungen und Effektivität (HSFK-Report 4/2007), Frankfurt/M. 2007. Der Aufsatz wurde für S+F insgesamt gestrafft und aktualisiert sowie um die Abschnitte 2.1 und 2.2 erweitert. Wir danken Prof. Dr. Michael Brzoska, Frau Susanne Bund, Axel Nitsche sowie zwei anonymen Gutachtern für ihre hilfreichen Kommentare.

2 Peter Rudolf, Sanktionen in der internationalen Politik. Zum Stand der Forschung (Studie der Stiftung Wissenschaft und Politik), Berlin 2006, 7-11. keit des Ziellandes und möglichst geringe wirtschaftliche Einbußen für die strafenden Staaten; ${ }^{3}$

4. ein differenziertes Verständnis von Wirksamkeit, das die ökonomische Wirkung von Sanktionen nicht mit der angestrebten Effektivität bei den politischen Zielen gleichsetzt. Selbst schwerste wirtschaftliche Schäden müssen nicht zu den erwünschten (außen-)politischen Verhaltensänderungen oder gar zu einer Destabilisierung der Regierung im Zielland führen.

Im Folgenden thematisieren wir diese Resultate der Sanktions-Literatur im Sinne der Forschungsfrage. Dies dient dazu, den Beitrag zu strukturieren. Die drei Erfolgsbedingungen sind insofern ein spezifisches Gliederungsprinzip, als wir sie bereits bei den unilateralen Zwangsmaßnahmen anwenden. Gleichzeitig gibt dieser Aufsatz darüber Aufschluss, inwieweit die drei Bedingungen für die Sanktionspolitik gegenüber dem Iran gelten.

\section{Vier Resultate der Sanktions-Forschung und der Fall Iran}

\subsection{Die begrenzte Wirkung unilateraler Maßnah- men: Die gescheiterte US-Politik}

»Unilaterale Sanktionen sind selten wirksam.«" Dieses Ergebnis, das sich aus der Sanktions-Literatur herauslesen lässt, verweist auf die Bedeutung der jeweiligen länderbezogenen Studien. Denn aus den im Einzelfall herausgearbeiteten Erklä-

3 Diese Trias von Erfolgsbedingungen lässt sich vor allem der nach wie vor umfangreichsten Sanktionsstudie von Hufbauer/Schott/Elliott, die in der Forschung Maßstäbe gesetzt hat, entnehmen (Gary Clyde Hufbauer/Jeffrey J. Schott/Kimberly Ann Elliott, Economic Sanctions Reconsidered: History and Current Policy, 2. Aufl., Washington, D.C. 1990, 91-115; siehe auch: Dies./ Barbara Oegg, Economic Sanctions Reconsidered, 3. Aufl., Washington, D.C. 2007,155-178)

4 Richard N. Haass, Conclusion: Lessons and Recommendations, in: Ders., Economic Sanctions and American Diplomacy, New York, N.Y 1998, 197-212, hier: 200 (im Original kursiv); siehe auch die in Anm. 12 angegebene Literatur. 
rungen über die wirtschaftliche Effektivität der Zwangsmaßnahmen lassen sich kaum generalisierende Schlussfolgerungen ableiten. Dies gilt auch für die US-Boykottpolitik gegenüber der Islamischen Republik. Die meisten empirischen Fallstudien analysieren mit unterschiedlichen qualitativen und quantitativen Methoden ${ }^{5}$ die Folgen des komplexen wie feinmaschigen Sanktionssystems der Vereinigten Staaten ${ }^{6}$ für die iranische Wirtschaft und das politische (Außen-)Verhalten Teherans. Die überwiegende Zahl der Analysen kommt zu dem Schluss, dass die seit der Geiselnahme der US-Botschaftsangehörigen im Jahre 1979 verhängten zahlreichen amerikanischen Wirtschafts- und Finanzmaßnahmen die iranische Volkswirtschaft geschwächt haben, wenn auch nicht in dem von den USA erhofften Ausmaß. Während der Clinton-Administration begann ein neues Kapitel in der Sanktionspolitik. Mit der Regierungsverordnung (Executive Order) Nr. 12959 vom 6. Mai 1995 wurde ein nahezu umfassendes Embargo für amerikanische Exporte und neue Investitionen im Iran verhängt. Der Iran-Libya Sanctions Act von 1996, auf Betreiben des Kongresses zustande gekommen und während der Amtszeit von George W. Bush zweimal verlängert, ging noch einen Schritt weiter: Dieses Gesetz wendet amerikanisches Recht extraterritorial an, indem es Sekundärsanktionen gegen ausländische Firmen oder Personen vorsieht, die mehr als 20 Mio. US-Dollar pro Jahr in den Öl- und Gassektor des Irans investieren. ${ }^{7}$ Allerdings haben die Präsidenten Clinton und Bush von ihrem im Gesetz verbrieften Recht Gebrauch gemacht Ausnahmegenehmigungen zu erteilen; damit haben sie es in der Praxis ausgehebelt.

Die negativen Folgen dieser restriktiven Politik machten sich für den Iran insbesondere in den ersten Jahren nach 1995 bemerkbar. ${ }^{8}$ Teheran sah sich gezwungen, Gegenmaßnahmen zu initiieren. Hierzu gehörten die Erweiterung der iranischen Produktpalette (größeres Gewicht auf den Nicht-Öl-Sektor) sowie die Gewinnung zahlreicher neuer Handels- und Investitionspartner. ${ }^{9}$ Das Regime musste allerdings häufig deren ungünstigere finanzielle Bedingungen akzeptieren. Die Sanktionen führten außerdem zur Verzögerung und Verteuerung maßgeblicher Investitionsprojekte. Dies machte die finanziellen Hauptbelastungen für die Islamische Republik aus. In den Jahren 1995 bis 2001 kosteten die unilateralen Sanktionen der Vereinigten Staaten den Iran verschiedenen Berechnungen zufolge jährlich zwischen 750 Mio. und 2,6 Mrd. US-Dollar. ${ }^{10}$ Die Zwangsmaßnahmen bewirkten insgesamt einen Verzögerungseffekt - keinen Stopp - für die maßgeblichen Projekte. »Die Sanktionen

5 Akbar E. Torbat, Impacts of the US Trade and Financial Sanctions on Iran, in: The World Economy 28, Nr. 3, 2005, 407-434, hier: 426. Torbats Übersicht fasst im Übrigen die Schätzungen mehrerer Studien zu den Kosten zusammen, die den USA und dem Iran aufgrund der unilateralen Sanktionen entstanden sind.

6 Einen (tabellarischen) Überblick geben: Hossein Alikhani, Sanctioning Iran: Anatomy of a Failed Policy, London 2000, 398-401; Hossein Askari/John Forrer/Hildy Teegen/Jiawen Yang, U.S. Economic Sanctions: Lessons from the Iranian Experience, in: Business Economics 36, Nr. 3, 2001, 7-19, hier: 10; Torbat (2005: 410f.).

7 Kinka Gerke, Die unilaterale Versuchung: Die Sanktionen der USA gegen die Handelspartner Kubas, Irans und Libyens und ihre Auswirkungen auf das Welthandelsregime (HSFK-Report 2/1997), Frankfurt/M. 1997, $29 \mathrm{f}$.

8 Meghan L. O'Sullivan: Shrewd Sanctions: Statecraft and State Sponsors of Terrorism, Washington, D.C. 2003, 66f.

9 Hooman Estelami, The Evolution of Iran's Reactive Measures to US Economic Sanctions, in: The Journal of Business in Developing Nations 2, 1998, www. ewp.rpi.edu/jbdn/jbdnv201.htm; letzter Zugriff: 20.11.2007; Jahangir Amuzegar, Adjusting to Sanctions, in: Foreign Affairs 76, Nr. 3, 1997, 31-41, hier: 34.

10 Torbat (2005: 426). haben der schon kranken iranischen Wirtschaft zusätzlichen Schmerz zugefügt, aber dieser ist sehr marginal gewesen. «11 Auch die politischen Wirkungen werden überwiegend als minimal bis »noch nicht bemerkbar ${ }^{12}$ bewertet.

Diese Einschätzung umfasst die angestrebten Verhaltensänderungen Teherans im Hinblick auf die Unterstützung des Terrorismus, im Nuklearbereich, beim Friedensprozess im Nahen Osten und bei den Menschenrechten im eigenen Lande. Der erwartete Mechanismus, dass sich der zunächst erzielte wirtschaftliche Schaden der US-Sanktionen politisch umsetzt, ist - so das Ergebnis praktisch aller Studien - nicht eingetreten. ${ }^{13}$ Dies ist u.a. auf die Möglichkeit gerade autoritärer Staaten zurückzuführen, systemstabilisierende Maßnahmen zu ergreifen, indem sie einen gesellschaftlichen Kohärenzeffekt zu erzeugen vermögen (z.B. über die partielle Steuerung der Medien).

Die Sanktionen hatten auch Folgen für die amerikanische Wirtschaft. Die Auswirkungen auf den Handel waren geringer als auf die Investitionsmöglichkeiten und Joint Ventures. ${ }^{14}$ Diese Verluste summieren sich nach Schätzungen für die Jahre 1995 bis 2001 auf rund eine Milliarde US-Dollar pro Jahr und liegen innerhalb der Schwankungsbreite der für Teheran berechneten Einbußen. ${ }^{15}$

Zusammengefasst: Der Faktor »internationale Geschlossenheit « hat bei den im Alleingang verhängten Strafmaßnahmen keine Rolle gespielt. Was die zweite Erfolgsbedingung anbelangt, so hat sich das Zielland Iran gegenüber den US-Sanktionen nur als begrenzt verwundbar erwiesen. Dies ist vor allem darauf zurückzuführen, dass insbesondere der Ölreichtum des Landes es den Teheraner Führungen ermöglicht hat, erfolgreich nach alternativen Importeuren zu suchen und das Produktionsspektrum zu erweitern. Der sanktionierende Staat USA hat beträchtliche Einbußen in Kauf genommen. Die amerikanischen Maßnahmen haben, ganz im Sinne des eingangs aufgeführten Resultats der Sanktions-Literatur, die Politik Teherans nicht beeinflussen können.

\subsection{Analytisch angemessen: Ein differenzierter Sanktionsbegriff}

Die Sanktions-Literatur wartet mit einer grundsätzlichen Unterscheidung der Instrumentarien auf, die den Umfang und die Adressaten betrifft. Im traditionellen Konzept umfassender Wirtschafts- und/oder Finanzbeschränkungen sollen Zwangsmaßnahmen indirekt wirken. Ihr Einsatz beruht auf der Annahme, dass sie im Zielland negative wirtschaftliche Folgen haben, die entweder Teile der Bevölkerung oder bestimmte

11 So Alikhani 2000: 408; ähnlich: Amuzegar (1997: 38); Estelami (1998).

12 Torbat (2005: 429).

13 O'Sullivan (2003: 88); ähnlich: Hossein G. Askari/John Forrer/Hildy Teegen/ Jiawen Yang, Economic Sanctions: Examining Their Philosophy and Efficacy, London 2003, 68f.; Ernest H. Preeg, Feeling Good or Doing Good with Sanctions: Unilateral Economic Sanctions and the U.S. National Interest, Washington, D.C. 1999, 74-78. »The overall net assessment of U.S. sanctions policy toward Iran since 1993 is thus predominantly negative. « (Preeg 1999: 77) - Auch ein Report des U.S. Government Accountability Office kommt zu einem skeptischen Fazit (U.S. Government Accountability Office, Iran Sanctions: Impact in Furthering U.S. Objectives Is Unclear and Should be Reviewed, Washington, D.C. 2007 [GAO-08-58]).

14 Askari et al. (2001: 18).

15 Askari et al. (2001: 18); Preeg (1999: 71). 
betroffene Gruppierungen (etwa Firmen) ${ }^{16}$ dazu bewegen, auf die herrschenden Eliten Druck auszuüben, damit diese ihrerseits ihr Verhalten im Sinne der strafenden Staaten ändern. Für die Eliten soll es sich damit irgendwann nicht mehr lohnen, ihre Politik beizubehalten. Diese Umwegstrategie nimmt das Leid der Zivilbevölkerung (bewusst) in Kauf. Das neue Konzept gezielter (»targeted «) oder kluger (»smart«) Sanktionen nimmt dagegen das Regime oder bestimmte Gruppen bzw. Güter/Technologien des Ziellandes direkt ins Visier. ${ }^{17}$ Die Zivilbevölkerung soll verschont bleiben. ${ }^{18}$

$\mathrm{Zu}$ den gezielten Strafmaßnahmen zählen: Waffenembargos, Reise-, Flug- und Transportbeschränkungen bzw. -verbote für bestimmte Gruppen und Personen sowie finanzpolitische Maßnahmen wie das Einfrieren von Auslandskonten und -kapital der Regierungen. Waffenembargos, von Kritikern als »moralisches Pflaster " bezeichnet, ${ }^{19}$ haben in der Forschung die Frage aufgeworfen, ob es sie nur auf dem Papier gibt. ${ }^{20}$ Cortright und Lopez zufolge ist der empirische Befund des von ihnen wesentlich mit entwickelten »smart sanctions «-Konzepts hier besonders enttäuschend: »Waffenembargos sind häufig auferlegt, aber selten durchgeführt worden. ${ }^{21}$ Reisesanktionen, anders als Rüstungsembargos ein neues Instrument der Zwangsdiplomatie, wandte der UN-Sicherheitsrat in den 1990er Jahren mehrfach mit unterschiedlicher Zielsetzung an - etwa, um die Unterstützung für den Terrorismus zu stoppen (Libyen und Afghanistan); um die Auslieferung von Terroristen zu erreichen (Libyen, Afghanistan) oder um die Einhaltung eines Friedensabkommens zu erzwingen (Angola/UNITA, Sierra Leone/RUF). Ein Beispiel für den Erfolg von Reiserestriktionen sind die Maßnahmen gegen Libyen. Ein über mehrere Jahre durchgehaltener umfassender Reise- und Flugboykott gegen Tripolis trug zu einer Verhaltensänderung der libyschen Machthaber bei.

Gezielte Finanzrestriktionen sind im UN-Rahmen in den 1990er Jahren in fünf Fällen beschlossen worden. Hierbei ist es notwendig, die Konten aufzuspüren und verlässlich zu identifizieren. Als Erfolgsbeispiel gilt Jugoslawien, wo die eingefrorene Summe von fast drei Milliarden US-Dollar enorme volkswirtschaftliche Folgen nach sich zog; ${ }^{22}$ die Finanzsanktionen trafen allerdings nicht nur Regierung und Elite. ${ }^{23}$ Aber auch dieses Instrument kann wirkungslos bleiben. In einer Reihe von Fällen erwies sich das Einfrieren von Auslandskonten als wenig effektiv, weil die betroffenen Personen den blockierten Geldfluss aus neuen Ein-

16 T. Clifton Morgan/Navin A. Bapat, Imposing Sanctions: States, Firms, and Economic Coercion, in: International Studies Review 5, Nr. 4, 2003, 65-79.

17 Michael Brzoska (Hrsg.), Smart Sanctions: The Next Steps. The Debate on Arms Embargoes and Travel Sanctions within the >Bonn-Berlin Process<, Baden-Baden 2001; David Cortright/George A. Lopez (Hrsg.), Smart Sanctions: Targeting Economic Statecraft, Boston, MA 2002; Peter Wallensteen/Carina Staibano (Hrsg.), International Sanctions: Between Words and Wars in the Global System, London 2005.

18 David Cortright/George A. Lopez, Introduction: Assessing Smart Sanctions: Lessons from the 1990s, in: Dies. (2002: 1-22, hier: 2).

19 Michael Brzoska, Putting More Teeth in UN Arms Embargoes, in: Cortright/ Lopez (2002: 125-143, hier: 125).

20 Loretta Bondi, Arms Embargos: In Name Only?, in: Cortright/Lopez (2002: 109-123, hier: 109).

21 Cortright/Lopez (2002: 1-22, hier: 14). - Zur Kritik am »smart sanctions«-Konzept siehe Daniel W. Drezner, How Smart are Smart Sanctions?, in: International Studies Review 5, Nr. 1, 2003, 107-110; Arne Tostensen/Beate Bull, Are Smart Sanctions Feasible?, in: World Politics 54, Nr. 3, 2002, 373-403.

22 Cortright/Lopez (2002: 1-22, hier: 11)

23 Anthonius W. de Vries, European Union Sanctions against the Federal Republic of Yugoslavia from 1998 to 2000: A Special Exercise in Targeting, in: Cortright/Lopez (2002: 87-108, hier: 104). nahmequellen mehr als ausgleichen konnten - etwa im Iran durch den Export von Öl (siehe 2.3.1).

Gezielte Sanktionen sind vorrangig auf eine symbolische ${ }^{24}$ und psychologische Wirkung ausgerichtet. Insbesondere Reiseverbote gelten als Ausdruck »internationaler Ächtung « und »diplomatischer Nichtanerkennung «. Die Verweigerung internationaler Legitimität soll schließlich in eine »Verweigerung innenpolitischer Legitimität « umschlagen. ${ }^{25}$ Im Hinblick auf gezielte Zwangsmaßnahmen steht die Geschlossenheit unter den sanktionierenden Staaten im Mittelpunkt, um einen maximalen Druck seitens der Weltöffentlichkeit ausüben zu können. Die Frage der Verwundbarkeit des gesamten Ziellandes stellt sich indes nicht, sondern bleibt auf das jeweilige Regime konzentriert. »Smart sanctions « können wegen ihrer primär symbolischen Wirkung in der Regel nur Teil einer umfassenderen außenpolitischen Strategie sein. Dieses Konzept muss in jedem Fall diplomatische Mittel, gegebenenfalls aber auch die Androhung militärischer Gewalt einschließen. ${ }^{26}$

\subsubsection{Die gezielten UN-Resolutionen und die brüchige Geschlossenheit der Sanktions- Allianz}

Die gegen die Nuklearpolitik des Iran gerichteten UN-Resolutionen 1737 und 1747 umfassen mit Reiserestriktionen und dem Einfrieren von Auslandskonten typische Elemente einer Strategie gezielter Sanktionen. Um wirksam sein zu können, ist daher die Geschlossenheit der internationalen Staatenwelt unter der Führung des Sicherheitsrats von entscheidender Bedeutung. Wie die langwierigen Verhandlungen sowie die verwässerten Resolutionsentwürfe zeigen, ist der Konsens im Sicherheitsrat jedoch insbesondere zwischen den Vereinigten Staaten einerseits sowie Russland und China andererseits brüchig. Es dauerte mehr als zwei Monate, bis die erste Resolution im Sicherheitsrat ausgehandelt war und verabschiedet werden konnte. ${ }^{27}$ Entschließung 1737 sieht drei Maßnahmen vor, wobei die Delegationen Russlands und Chinas die strikteren Vorschläge der westlichen Mitglieder des UN-Sicherheitsrats entschärften. So wurde aus einem umfassenden Ausfuhrverbot für Materialien und Technologien im Nuklear- und Raketenbereich ein Verbot, das nur spezifisch aufgeführte Güter betrifft; die Anzahl der in diesen Sektoren tätigen Iraner, deren Auslandsguthaben eingefroren wurde, beschränkte sich auf ein Minimum; und die ursprünglich vorgesehenen Einreiseverbote für eine Reihe von iranischen Militärs und Wissenschaftlern wandelten Moskau und Beijing in Reisebeschränkungen um.

Entschließung 1747 vom 24. März 2007 folgte im Wesentlichen den Vorgaben der Vorgängerresolution. Die drei genannten Sanktionselemente - spezifische Exportverbote, Finanzsanktionen und Reisebeschränkungen - wurden verschärft und ihr Einzugsbereich erweitert. Mit der Sepah Bank war erstmals ein

24 David A. Baldwin, Economic Statecraft, Princeton, NJ 1985, 115-144.

25 Richard W. Conroy, The UN Experience with Travel Sanctions: Selected Cases and Conclusions, in: Cortright/Lopez (2002: 145-169, hier: 163).

26 Kimberly Ann Elliott, Analyzing the Effects of Targeted Sanctions, in: Cortright/Lopez (2002: 171-182, hier: 175).

27 International Herald Tribune, 9.11.2006. 
großes iranisches Geldinstitut von den Maßnahmen betroffen. Trotz des im Sicherheitsrat erzielten Kompromisses ist die Umsetzung der Resolutionen nicht gewährleistet. Die wirtschaftlichen Interessen, Schlupflöcher zu finden und auszunutzen, sind groß, vor allem in Russland. Moskau rechnet offenbar mit bilateralen Verträgen bei Rüstungsgütern und Kernkraftwerken in Milliardenhöhe. ${ }^{28}$ Allein die vereinbarte russische Lieferungen von 29 Tor-M 1-Raketenabwehrsystemen soll einen Ertrag von 700 Mio. bis 1,4 Mrd. US-Dollar erbringen. ${ }^{29}$ Derartige Aktivitäten russischer Firmen sind in der Vergangenheit ein ständiger Streitpunkt zwischen Moskau und Washington gewesen. Das Gleiche gilt für die Iran-Politik Beijings, die maßgeblich durch den stetig wachsenden Energiebedarf im »Reich der Mitte bestimmt wird.

Die unterschiedlichen wirtschaftlichen Interessen sind ihrerseits in divergierende außenpolitische Konzepte der maßgeblichen Akteure eingebettet. So setzen Russland und China grundsätzlich auf Diplomatie und Verhandlungen; eine militärische »Lösung « schließen sie aus. Sie sind bestrebt, die wirtschaftlichen Kosten zu erhöhen, um Teheran an den Verhandlungstisch zurückzuholen, aber nicht um jeden Preis. Mit ihrem Engagement in der Iran-Frage zeigen beide Staaten, dass sie als externe Akteure in der gesamten Region eine bedeutsame Rolle spielen wollen. Weltordnungs- und Regionalpolitik heißt für sie, den Nahen Osten/Persischen Golf nicht allein den Vereinigten Staaten zu überlassen. Im außen- und sicherheitspolitischen Kalkül Washingtons sind Zwangsmaßnahmen hingegen nicht nur mit der Hoffnung verbunden, dass der internationale Druck auf Teheran zu einer Verhaltensänderung im Nuklearkonflikt führt. Die Unterstützung des Terrorismus, die Ablehnung des Nahost-Friedensprozesses, Irans Aspirationen auf regionale Vorherrschaft und die Unterdrückung des eigenen Volkes gehören ebenfalls zur »Totalität der Bedrohung «. ${ }^{30}$ Washington geht es bislang nicht um die Einbindung, sondern um die verschärfte internationale Isolation der Islamischen Republik.

Das Ziel eines notfalls gewaltsam durchgeführten »regime change « in Teheran nahm die Bush-Administration selbst nach der Veröffentlichung des politisch brisanten National Intelligence Estimate vom November 2007 nicht zurück. ${ }^{31}$ Alle 16 Geheimdienste waren darin zu der Einschätzung gelangt, dass der Iran seit 2003 kein Atomwaffenprogramm mehr unterhalte. ${ }^{32}$ Dass die Diplomatie zunächst Vorrang hat, aber erfolgreich bei der Lösung des Atomkonflikts sein muss, damit es nicht zur militärischen Konfrontation kommt, steht im Einklang mit dem umfassenden außenpolitischen Konzept Washingtons, das in der »National Security Strategy« der US-Administration seinen Ausdruck gefunden hat. Das hat England, Frankreich und Deutschland aber nicht davon abgehalten, im Kontext der UN-Resolutionen 1737 und 1747 eine harte Haltung ein-

28 Victor Mizin, The Russian-Iran Nuclear Connection and U.S. Policy Options, in: Middle East Review of International Affairs 8, Nr. 1, 2004, 71-85, hier: 76.

29 GlobalSecurity.org, www.globalsecurity.org/military/world/iran/airforce. htm; letzter Zugriff: 12.6.2007; bzw. Eurasia Daily Monitor, 8.1.2007.

30 So Nicholas Burns, in: 109/2 U.S. Congress, House of Representatives, Committee on International Relations: U.S. Policy Towards Iran: Next Steps, Hearing, 8.3.2006 (unautorisiertes Transkript), 15

31 International Herald Tribune, 5.12.2007.

32 Office of the Director of National Intelligence, National Intelligence Estimate, Iran: Nuclear Intentions and Capabilities, Washington, D.C. 2007. zunehmen. Derzeit lässt sich festhalten, dass die Europäische Union im Umfeld des Außenbeauftragten Solana konzeptionell wie auch in der Praxis eine Doppelstrategie befürwortet, die neben Zwangsmaßnahmen das Angebot von Verhandlungen einschließt. ${ }^{33}$ Kurzum, die erste Erfolgsbedingung - die Geschlossenheit innerhalb der Sanktions-Allianz - ist bisher von ökonomischen Interessen und außenpolitischen Divergenzen merklich beeinträchtigt worden.

\subsubsection{Die gegenwärtige US-Kampagne zur um- fassenden Blockade des Irans}

Die Auffassung, dass umfassende Sanktionen mit beträchtlichen negativen Folgen ethisch nicht mehr zu rechtfertigen seien, leitet rhetorisch auch die Bush-Administration bei ihrer multilateralen UN-Politik an. In der Praxis hält sie jedoch die Maßnahmen der Vereinten Nationen keinesfalls für ausreichend. Deshalb hat die Vormacht USA spätestens seit Sommer 2006 unilateral Initiativen in Gang gebracht, die sie bilateral durchführt. Sie ergänzen die Zwangsmittel im UN-Rahmen und erweisen sich als das Kernstück der amerikanischen Sanktionspolitik gegenüber Teheran. Vor allem das US-Finanzministerium betreibt eine stille wie gezielte Reisediplomatie. Führende Repräsentanten sprechen bei den Spitzenvertretern der Banken und Wirtschaftsunternehmen in befreundeten Staaten vor. USFinanzminister Henry Paulson und seine Top-Beamten fordern dazu auf, alle Investitionstätigkeiten im Iran zu beenden. ${ }^{34}$ Die amerikanischen Emissäre übten zumindest auf einige ihrer Gesprächspartner so starken Druck aus, dass sich diese hinter vorgehaltener Hand über »Erpressung « beklagten. ${ }^{35}$ Und dennoch kann bislang kaum die Rede davon sein, dass die Vormacht Vereinigte Staaten die angestrebte Geschlossenheit unter den kontaktierten Akteuren erreicht hat: Viele Geldinstitute, die die Dollargeschäfte mit der Islamischen Republik eingestellt oder reduziert haben, lassen offen, in welchem Ausmaß sie weiterhin in anderen Währungen mit Teheran Finanzbeziehungen unterhalten. Lediglich drei größere europäische Banken zogen sich im Jahre 2006 nach Aussage von Nicholas Burns, dem damaligen Architekten der Iran-Politik im US-Außenministerium, vollständig aus dem Geschäft mit der Islamischen Republik zurück. ${ }^{36}$

Aus Sicht der US-Regierung stellen ihre Maßnahmen eine unerlässliche Ergänzung zu den primär symbolischen UN-Sanktionen dar. Tatsächlich aber unterminieren sie die internationale Geschlossenheit. Ihren Willen zum Alleingang hat die Regierung Bush mehrfach deutlich gemacht. So erklärte sie im Oktober 2006 im Rahmen eines Sanktionspakets mit der rund 2.500 Kämpfer umfassenden Al-Kuds-Brigade erstmals eine staatliche

33 Financial Times Deutschland, 13.2.2007.

34 Stuart Levey, Undersecretary of Treasury for Terrorism and Financial Intelligence, in: 110/1 U.S. Congress, Senate, Committee on Banking, Housing, and Urban Affairs: Minimizing Potential Threats From Iran: Assessing the Effectiveness of Current U.S. Sanctions on Iran, Hearing, 21.3.2007 (unautorisiertes Transkript), 40.

35 Spiegel Online, www.spiegel.de/international/business/0,1518,497319,00. html, 30.7.2007.

36 110/1 U.S. Congress, House of Representatives, Committee on Foreign Affairs: The Iranian Challenge, Hearing, 6.3.2007 (unautorisiertes Transkript), 20; siehe auch: Neue Zürcher Zeitung, 27./28.10.2007; The Guardian, 9.11.2007. 
Militärformation zu einer Terrorgruppe. ${ }^{37}$ Eine andere Maßnahme des Sanktionspakets läuft für alle amerikanischen Banken auf das Verbot hinaus, selbst indirekt in Dollargeschäfte vor allem mit drei großen iranischen Banken (Melli, Mellat und Saderat) involviert zu sein. ${ }^{38}$ Auf diesem Wege sollen die iranischen Geldinstitute aus dem globalen Finanzsystem ausgeschlossen werden. ${ }^{39}$ Mit der Ausweitung der Finanzsanktionen auf die großen iranischen Banken sprengt die amerikanische Regierung den Ansatz gezielter Maßnahmen gegen Teheran. Denn damit wird auch die iranische Bevölkerung getroffen. Der differenzierte Sanktionsbegriff der Forschung erweist sich hier als notwendig, weil er nicht nur gezielte, sondern auch umfangreiche Maßnahmen thematisiert.

\subsection{Analytisches Kernstück: Die wesentlichen Er- folgsbedingungen von Zwangsmaßnahmen}

Von den drei erwähnten Erfolgskriterien (siehe Kapitel 1) haben wir den Faktor Geschlossenheit bereits behandelt (siehe 2.2.1 und 2.2.2). Im Folgenden konzentrieren wir uns auf die wirtschaftliche Verwundbarkeit des Irans und auf die Kosten für die sanktionierenden Staaten; hiermit geht jeweils eine knappe Einschätzung des Sanktionspotenzials einher.

\subsubsection{Die Verwundbarkeit des Irans}

Die Volkswirtschaft und der Energiebereich der Islamischen Republik weisen die folgenden vier zentralen Schwachstellen auf:

Die Abhängigkeit der Öl- und Gasindustrie von ausländischen Investitionen: Ist ein internationaler Stopp wirksam? Trotz seiner enormen Öl- und Gasreserven steht das Land vor dem grundlegenden Problem, in seine veraltete Öl- und Gasindustrie enorme Summen investieren zu müssen, um die gegenwärtige Förderrate in beiden Sektoren aufrechtzuerhalten oder gar zu erhöhen. Ohne gewaltige Investitionen aus dem Ausland sind all diese für die iranische Volkswirtschaft wesentlichen Aufgaben nicht zu bewältigen. Die Rolle asiatischer Länder mit drastisch steigendem Energiebedarf - allen voran China, Indien und Malaysia - dürfte die Wirkung von Investitionsbeschränkungen westlicher Staaten erheblich einschränken. Dies setzt natürlich voraus, dass jene asiatischen Länder bei ihrem derzeitigen Kurs bleiben und weiterhin als wichtige Investitionspartner der Islamischen Republik agieren. ${ }^{40}$ Ein ausschließlich von europäischen Ländern durchgeführter Investitionsstopp würde, selbst wenn er sich realisieren ließe, die iranischen Fördermengen bestenfalls in einigen Jahren beeinträchtigen, wie selbst Befürworter einer strikten Sanktionspolitik einräumen. ${ }^{41}$

37 Financial Times Deutschland, 26.10.2007.

38 Bundesagentur für Außenwirtschaft, US-Sanktionen gegen iranische Banken (Typoskript), 2007.

39 The New York Times, 4.11.2007.

40 Allein Beijing verhandelt derzeit mit Teheran über langfristige Energieprojekte, deren finanzielles Volumen offenbar im dreistelligen Milliardenbereich liegt.

41 Patrick Clawson/Michael Eisenstadt, Forcing Hard Choices on Tehran: Raising the Costs of Iran's Nuclear Program (Policy Focus No. 62), Washington, D.C. 2006, 16.
Die steigende Nachfrage nach raffinierten Ölerzeugnissen: Zwangsmaßnahmen als forcierendes Element? Knapp über 40 Prozent der im Iran verbrauchten Ölprodukte werden aus dem Ausland importiert. Besonders brisant und politisch potenziell explosiv ist dabei die Einfuhr von Benzin. Selbst nach der Fertigstellung geplanter neuer Raffineriekapazitäten wird das Regime die unzureichende Benzinversorgung nicht in den Griff bekommen. ${ }^{42}$ Die iranische Regierung sah sich sogar gezwungen, den Kraftstoffverbrauch zu rationieren. Dies birgt die Gefahr sozialer und politischer Proteste. Um eine solche Entwicklung zu vermeiden, muss Teheran bestrebt sein, den Öl- und Gaspreis durch verstärkte Subventionierungen möglichst niedrig zu halten. Für die Teheraner Führung ist ein hoher Energiepreis dabei von Vorteil (siehe unten). Solange vor allem das Öl auf dem Weltmarkt zu Rekordsummen gehandelt wird, dürfte sich kein Sanktionspotenzial externer Akteure ergeben.

Die Abhängigkeit von den Einnahmen aus dem Rohölexport: Ein internationaler Ölboykott als zweischneidiges Schwert? Ohne Ölund Gasausfuhr ist die iranische Ökonomie über kurz oder lang nicht lebensfähig. Der Anteil beider Stoffe am gesamten Exportvolumen lag 2004/05 bei rund 80 Prozent. Die Monostruktur der Ausfuhr, also die hochgradige Abhängigkeit des Irans von Öl- und Gasexporten, eröffnet auf den ersten Blick ein großes Sanktionspotenzial. Andererseits könnte Teheran einen Ausfuhrstopp bei seinem »schwarzen Gold « verfügen und so einen drastischen Anstieg des Ölpreises herbeiführen. Ein Ölboykott, ob durch die Importländer oder den Iran veranlasst, hätte für beide Seiten unabsehbare Folgen. Fachleute vermuten daher, dass keine Seite ein Interesse daran hat, die Ölwaffe einzusetzen. ${ }^{43}$

Die gravierenden gesamtwirtschaftlichen Probleme: Sanktionen als verschärfender Faktor? Die iranische Wirtschaft ist seit der Wahl von Ahmadinedschad zum Staatspräsidenten mit gravierenden strukturellen Problemen zum Teil verstärkt konfrontiert. Neben der Monostruktur der Wirtschaft sind die Kaufkraftverluste durch Inflation, die wachsende Kapitalflucht, die um sich greifende Korruption sowie die hohe Arbeitslosigkeit (für 2005/06 offiziell: 14,6 Prozent; inoffiziell: über 50 Prozent) zunehmend drängende Probleme. Als mächtigster Handelsblock hat die EU eine potenziell starke Position, wirtschaftlichen Druck auf den Iran auszuüben. ${ }^{44}$ Teheran hat sich aber bereits auf solche Maßnahmen vorbereitet und Gegen- und Umwegstrategien entwickelt; hierzu gehören der Aufbau von Lagern für wichtige Güter sowie der Ausbau von Handelswegen (insbesondere über Dubai) ${ }^{45}$ Die Wirksamkeit von Sanktionen ist vor diesem Hintergrund schwer zu beurteilen.

Zusammengefasst: Jeder einzelne der vier genannten Faktoren kann eine beträchtliche Verschlechterung der wirtschaftlichen Lage im Iran erzeugen. Es stellt sich jedoch die Frage, wie wahrscheinlich es ist, dass es der Sanktions-Allianz gelingt, eine derartige Wirkung zu erzielen. In jedem Fall müsste die Koalition der bestrafenden Staaten über den Rahmen der bisher in den

42 Iran Daily, www.iran-daily.com/1383/2165/html/focus.htm, 16.12.2004.

43 Clawson/Eisenstadt (2006: 17).

44 George Perkovich/Silvia Manzanero, Plan B: Using Sanctions to End Iran's Nuclear Program, in: Arms Control Today 34, Nr. 4, 20-25, hier: 24.

45 Wirtschaftskammer Österreich, Iran-Newsletter Nr. 15 (Typoskript), 2006, 
UN vereinbarten »smart sanctions « beträchtlich hinausgehen. Die erforderliche Geschlossenheit der sanktionierenden Staaten müsste sich auf den gesamten Handel und die Investitionen im Iran erstrecken. Hinzu kommt, dass vor allem die wichtigsten Partner des Irans im Falle von umfangreichen Zwangsmaßnahmen bereit sein müssten, beträchtliche wirtschaftliche Kosten zu tragen.

\subsubsection{Die (drohenden) Kosten für die sanktionie- renden Staaten}

Für den angenommenen Fall eines internationalen Ausfuhrstopps, der das gesamte Warenspektrum in den Iran umfassen würde, ergäben sich die Kosten für die boykottierenden Staaten gemäß ihres Handelsvolumens mit Teheran. Die Vereinigten Arabischen Emirate rangieren hier auf dem ersten Platz, wobei ihre Sonderstellung darin besteht, dass sie die Drehscheibe für Firmen sind, die nicht direkt in den Iran liefern. Deutschland ist der zweitwichtigste Handelspartner der Islamischen Republik. In den Jahren 2005/2006 belief sich sein Exportvolumen auf rund vier Mrd. US-Dollar. ${ }^{46}$ Sowohl aufgrund der amerikanischen De-Investmentkampagne als auch der unvermindert kompromisslosen Haltung Teherans sind nicht nur in Berlin, sondern auch in Brüssel Diskussionen über verschärfte Sanktionen entbrannt, die über die UN-Zwangsmaßnahmen hinausgehen sollen. Die Positionen sind in dieser Frage auf Länderund Europaebene jedoch uneinheitlich. Vor allem zwischen den Regierungen in Italien und Frankreich - letztere drängt auf verschärfte Sanktionen - ist es zu Kontroversen über das Ausmaß zukünftiger Exportbeschränkungen gekommen. ${ }^{47}$ In Deutschland ist die Kritik der Wirtschaftsverbände unüberhörbar. ${ }^{48}$ Immerhin war bei den deutschen Exporten in den Iran in den ersten sieben Monaten des Jahres 2007 ein Rückgang von 18 Prozent gegenüber dem vergleichbaren Zeitraum von 2006 zu verzeichnen. ${ }^{49}$

Derzeit lässt sich nicht sagen, ob die europäischen Staaten ein nachhaltiges Embargo gegenüber der Islamischen Republik auf breiter Basis beschließen und durchführen werden. Damit ist aus heutiger Sicht auch unklar, in welchem Ausmaß die EUMitglieder bereit sind, mögliche wirtschaftliche Einbußen zu akzeptieren. Für Indien und China, zwei Staaten mit wachsenden Volkswirtschaften und zunehmendem Energiebedarf, gilt das indes nicht. Sie hätten erhebliche Kosten zu tragen und stehen deshalb drastischen Sanktionen äußerst skeptisch gegenüber. Sollte es zu einem europäischen Boykott gegenüber dem Iran kommen, könnten beide Länder nicht nur ausbleibende Handelsgüter, sondern auch Investitionen mittel- und langfristig ausgleichen.

\footnotetext{
46 Bundesagentur für Außenwirtschaft, Iran-Wirtschaftsdaten kompakt (Typoskript), 2007, 4

47 International Herald Tribune, 9.10.2007

48 Die Welt, 13.11.2007.

49 Bundesagentur für Außenwirtschaft, Neue Sanktionen gegen den Iran (Typoskript), 2007. - Dem gegenüber erklärte der deutsche Botschafter im Iran, Herbert Honsowitz, dass deutsche Exporte bisher nicht beeinträchtigt wurden, weil sie die Islamische Republik hauptsächlich über die Vereinigten Arabischen Emirate erreichten (Die Welt, 23.1.2008)
}

\section{Fazit und Ausblick: Unter welchen Bedin- gungen können Sanktionen eine Nuklear- macht Iran verhindern?}

Für die zusammenfassende Einschätzung der Zwangsmaßnahmen, und zwar sowohl der »smart sanctions « des UN-Sicherheitsrats als auch der von Washington initiierten umfassenden Sanktionen, ist die unterteilte Wirkung in eine wirtschaftliche und politische Dimension (siehe Kapitel 1) bedeutsam. Beide Aspekte können zum gegenwärtigen Zeitpunkt allein deshalb nur vorläufig beantwortet werden, weil die unterschiedlichen Maßnahmen erst angelaufen sind.

Die amerikanische De-Investmentkampagne hat in der islamischen Republik eine "gewisse « wirtschaftliche und politische Wirkung erzielt. Zu dieser vagen Einschätzung (»It's having some effect.«) kam US-Unterstaatssekretär Stuart Levey im März 2007 in seiner betont zurückhaltenden Erfolgsbilanz. In wirtschaftlicher Hinsicht ist diese Zurückhaltung insofern gerechtfertigt, als insbesondere jene drei iranischen Banken, welche die Bush-Administration bei ihrem unilateral beschlossenen Sanktionspaket vom Oktober 2007 ins Visier nahm, ihre Geschäfte, soweit bekannt, nach wie vor ungehindert tätigen können - wenn auch zu erhöhten Kosten. ${ }^{50}$

Als Indikator für die politische Wirkung führte Levey die »interne Debatte im Iran« darüber an, in welche Richtung das Land gehen solle. ${ }^{51}$ Einige moderate Kräfte, wie der frühere Chef-Unterhändler in den Atomgesprächen, Hassan Rowhani, haben in der Tat die konfrontative iranische Außenpolitik kritisiert. ${ }^{52}$ Bis jetzt gibt es jedoch keine Signale von Seiten der Teheraner Führung, die auf die Einstellung des Urananreicherungsprogramms schließen lassen. Vielmehr hat das Regime seine Kapazitäten in diesem Bereich beharrlich ausgebaut. ${ }^{53}$ Die Ernennung des Ahmadinedschad-Vertrauten Said Dschalili zum neuen ChefUnterhändler Teherans deutet darauf hin, dass die Hardliner ihre kompromisslose Position verstärken konnten. Diese Entwicklung mag ein Grund dafür sein, dass Stuart Levey seine vorsichtig positive Einschätzung über die Wirkung der Sanktionen in späteren Ausführungen vor dem Kongress im Oktober 2007 nicht wiederholt hat. ${ }^{54}$ Vor diesem Hintergrund lässt sich festhalten, dass auch die gezielten UN-Sanktionen ihre erhoffte Wirkung bislang nicht entfaltet haben.

Wie wahrscheinlich ist auf absehbare Zeit ein Wandel der unnachgiebigen Position Teherans im Atomstreit? Die Sanktions-Forschung offeriert keine eindeutigen Prognosen, wohl aber hat sie drei Aspekte herausgearbeitet, die Aufschluss über den künftigen Weg Teherans geben könnten. Der erste Aspekt bezieht sich auf die von Hufbauer/Schott/Elliott formulierte Faustformel `Je anspruchsvoller das Ziel, desto geringer die Ef-

50 The Boston Globe, www.boston.com/news/world/articles/2007/12/16/bush_ effort_to_sanction_iran_banks_runs_into_resistance, 16.12.2007.

51 110/1 U.S. Congress, Senate, Committee on Banking, Housing, and Urban Affairs: Minimizing Potential Threats From Iran: Assessing the Effectiveness of Current U.S. Sanctions on Iran, Hearing, 21.3.2007 (unautorisiertes Transkript), 14,40 .

52 The Associated Press, 12.11.2007.

53 International Herald Tribune, 30.11.2007.

54 Special on-the-Record-Briefing on Iran with R. Nicholas Burns, Undersecretary of State for Political Affairs, and Undersecretary of Treasury for Terrorism and Financial Intelligence, Stuart Levey (as Released by the State Department), 25.10.2007, Washington, D.C. (Transkript). 
fektivität der Zwangsinstrumente $\prec .{ }^{55}$ Die politische Zielsetzung der UN-Resolutionen, eine Verhaltensänderung Teherans bei der Urananreicherung zu bewirken, ist in der Tat sehr weitgehend. Die Anreicherung wird inzwischen als ein Anliegen von zentraler nationaler Bedeutung gesehen; auch in der sonst in mehrere Fraktionen gespaltenen Elite im Iran ist sie unumstritten. Der zweite Aspekt betrifft die Frage, inwieweit die Sanktionen die Motivationen beeinflussen können, die hinter Teherans nuklearen Ambitionen stehen. Hierzu gehören hegemonialer Ehrgeiz, regionaler Status, das Prestige, eine komplexe Technologie zu meistern und Sicherheitsinteressen. Sie alle werden mit den wirtschaftlichen Zwangsmaßnahmen nicht angesprochen. Und drittens verweist die Forschung auf die Relevanz von Sanktionen für die Machterhaltung des Regimes. Bislang hält der Druck von außen Elite und Bevölkerung (Regimekritiker eingeschlossen) zusammen. Ein nachhaltiger »rally-aroundthe-flag«-Effekt könnte sich aus heutiger Sicht also durchaus einstellen - und damit genau das Gegenteil von dem bewirken,

55 Hufbauer/Schott/Elliott (1990: 38); siehe auch: Dies/Barbara Oegg, Economic Sanctions Reconsidered, 3. Aufl., Washington, D.C. 2007, 159. was die Sanktions-Allianz politisch beabsichtigt. Diese Auffassung teilen selbst führende Vertreter der Regierung Bush. ${ }^{56}$

Zusammengefasst: Die Sanktions-Forschung kann weder eindeutige noch allgemeingültige Voraussagen für den Einsatz von wirtschafts- und finanzpolitischen Zwangsmaßnahmen treffen. ${ }^{57}$ Dennoch liegt mit den vier vergleichsweise gesicherten Ergebnissen ein analytisches Instrumentarium vor, das sich auf den »Fall Iran « produktiv anwenden lässt. Empirisch spricht derzeit wenig dafür, dass es gelingen wird, den Iran durch Sanktionen zu einem Verzicht auf die Urananreicherung zu drängen. Ein Erfolg setzt mindestens voraus, dass sowohl die Europäische Union als auch China, Russland und Indien ihre Wirtschaftsbeziehungen mit der Islamischen Republik beträchtlich und nachhaltig einschränken. Die oben genannten drei Aspekte dürften nicht nur dazu dienen, die Entwicklungen in der Islamischen Republik zu beobachten und einzuschätzen. Diese systematische, vergleichende Analyse von Zielländern eröffnet der empirischen Sanktions-Forschung jenseits der einzelnen Fallstudien vielmehr ein weites Feld.

56 So R. Nicholas Burns, in: 110/1 U.S. Congress, Senate, Committee on Banking, Housing, and Urban Affairs: Minimizing Potential Threats From Iran: Assessing the Effectiveness of Current U.S. Sanctions on Iran, Hearing, 21.3.2007 (unautorisiertes Transkript), 23.

57 Rudolf (2006: 6).

\title{
Nuclear Non-Proliferation as a Learning Case for Civil Society Legitimacy
}

\author{
Claudia Kissling ${ }^{1}$
}

\begin{abstract}
Recent years have seen growing scholarly interest in civil society legitimacy. The present article proposes four normative criteria, namely: independence, transparency, participation, and inclusion, that could be applied to start assessing civil society legitimacy empirically. It does so with reference to the non-proliferation regime, analysing three advocacy NGOs active during the Nuclear Non-Proliferation Treaty's review processes - the MPI, the Friedenswerkstatt, and the IPPNW. The results give a fairly good picture of CSO legitimacy, but show that what seems to matter most in this regard is organizational culture, rather than organizational structure.
\end{abstract}

Keywords: civil society, legitimacy, nuclear non-proliferation

\section{Introduction}

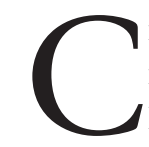
ivil Society Organizations (CSOs) have sparked a growing interest among scholars and practitioners over the last years (see e.g. Steffek, Kissling et al. 2007). After a period of favourable contemplation, however, civil society

1 Peer-reviewed article. Dr Claudia Kissling was a Research Fellow at the Collaborative Research Center `Transformations of the State at the University of Bremen. actions meanwhile meet with increasing criticism. CSOs are perceived as the expression of an emancipatory, even resistant civil society (Cox 1999), or, from an opposite standpoint, as servants to (neo)liberal capitalism (for both, see Amoore and Langley 2004; Goonewardena and Rankin 2004), or even as a postmodern amalgam of various, including religious, groupings (Kaldor 2003; Courville 2006: 272). The more CSOs assume power, and the more successful they are in bringing the issues of democracy, accountability and legitimacy of global 